\title{
Early cardiac effects of contemporary radiation therapy in breast cancer patients
}

Short running title: Early cardiac changes in breast cancer radiation

Suparna C. Clasen, $\mathrm{MD}^{\ddagger}$, Haochang Shou, $\mathrm{PhD}^{\#}$, Gary Freedman, $\mathrm{MD}^{\dagger, \neq}$, John P. Plastaras $\mathrm{MD}, \mathrm{PhD}^{\dagger, \neq}$, Neil K. Taunk, MD, $\mathrm{MS}^{\dagger}$, Boon-Keng Kevin Teo, $\mathrm{PhD}^{\dagger}$, Amanda M. Smith, BA, $M^{*}$, Biniyam G. Demissei MD, MSc, PhD*, Bonnie Ky, MD, MSCE*\#

${ }^{\ddagger}$ Krannert Institute of Cardiology and Division of Cardiology, Department of Medicine, Indiana University School of Medicine, Indiana University, Indianapolis, Indiana;

*Department of Medicine, Division of Cardiology, Perelman School of Medicine at the University of Pennsylvania, Philadelphia, Pennsylvania;

${ }^{\dagger}$ Department of Radiation Oncology, Perelman School of Medicine at the University of Pennsylvania, Philadelphia, Pennsylvania;

${ }^{\ddagger}$ Abramson Cancer Center, Perelman School of Medicine at the University of Pennsylvania, Philadelphia, Pennsylvania;

"Department of Biostatistics, Epidemiology \& Informatics, Perelman School of Medicine at the University of Pennsylvania, Philadelphia, Pennsylvania

\section{Corresponding Author:}

Bonnie Ky, MD, MSCE

3400 Civic Center Boulevard

Smilow Center for Translational Research

Philadelphia, PA 18194

2155736606

bonnie.ky@pennmedicine.upenn.edu

This is the author's manuscript of the article published in final edited form as:

Clasen, S. C., Shou, H., Freedman, G., Plastaras, J. P., Taunk, N. K., Teo, B.-K. K., Smith, A. M., Demissei, B. G., \& Ky, B. (2020). Early Cardiac Effects of Contemporary Radiation Therapy in Patients With Breast Cancer. International Journal of Radiation Oncology, Biology, Physics. https://doi.org/10.1016/j.jrobp.2020.12.008 
Authors Responsible for Statistical Analysis:

Suparna C. Clasen - 60355775081801 N. Senate Boulevard, Suite 400 Indianapolis IN 46202 scclasen@iu.edu

Haochang Shou - 215573 6534, 219 Blockley Hall, 423 Guardian Drive, Philadelphia, PA 19104

hshou@pennmedicine.upenn.edu

Conflict of Interest: SCC ${ }^{\ddagger}$ - immediate family member at Eli Lilly, no direct conflicts of interest. $\mathrm{NKT}^{\dagger}$ - speaking and advisory board fees from Sensus Healthcare, Varian Medical Systems, and RefleXion Medical, no direct conflicts of interest

\section{Funding:}

This work was supported by an Abramson Cancer Center and Radiation Oncology Pilot Grant Award (Ky), by R01 HL 118018 (Ky), by T32 HL 007891 grant (Clasen). 


\section{Early Cardiac Effects of Contemporary Radiation Therapy in Breast Cancer Patients}

\section{ABSTRACT}

PURPOSE: To characterize the early changes in echocardiographic-derived measures of cardiac function with contemporary radiation therapy $(\mathrm{RT})$ in breast cancer and determine the associations with radiation dose-volume metrics including mean heart dose (MHD). METHODS AND MATERIALS: In a prospective longitudinal cohort study of 86 breast cancer patients treated with photon or proton thoracic $\mathrm{RT}$, clinical and echocardiographic data were assessed at three time points: within 4 weeks before RT initiation (T0); within 3 days prior to 6 weeks after the end of RT (T1), and 5-9 months after RT completion (T2). Associations between MHD and echocardiographic-derived measures of cardiac function were assessed using generalized estimating equations to define the acute (T0 to T1) and subacute (T0 to T2) changes in cardiac function.

RESULTS: The median (IQR) estimates of MHD ranged from 139 cGy (99-249 cGy). In evaluating the acute changes in LVEF from T0 to T1, and in accounting for the time from RT, age, race, pre-existing cardiovascular disease, and an interaction term with anthracycline and/or trastuzumab exposure and MHD, there was a modest decrease in LVEF of borderline significance $(0.22 \%, 95 \% \mathrm{Cl}-0.44 \%, 0.01 \% ; p=0.06$, per 30 -day interval for every $100 \mathrm{cGy}$ increase of MHD). Similarly, there was a modest worsening in longitudinal strain $(0.19 \%, 95 \%$ $\mathrm{Cl}-0.01 \%, 0.39 \% ; p=0.06$ ), per 30-day interval for each $100 \mathrm{cGy}$ increase in MHD. We did not find significant associations between MHD and changes in circumferential strain or diastolic function.

CONCLUSION: With modern radiation planning techniques, there are very modest subclinical changes in measures of cardiac function in the short-term. Longer-term follow-up studies are needed to determine if these early changes are associated with the development of overt cardiac disease. 


\section{INTRODUCTION}

Radiation therapy $(\mathrm{RT})$ is fundamental to breast cancer treatment, resulting in important reductions in disease morbidity and mortality (1). However, thoracic RT can potentially lead to increased cardiovascular morbidity and mortality. Historical data from 1954 to 1984 suggest that those treated with $\mathrm{RT}$ had a 1.76 fold $(95 \% \mathrm{Cl} 1.34,2.31)$ increased risk of cardiovascular death (2). A seminal population-based case-control study by Darby et al. demonstrated that the risk of a major coronary event increased proportionally with mean heart dose (MHD) without an apparent threshold to the risk (3). These studies have defined the late cardiac effects of RT, which may present years after RT exposure (4-6). The cardiac effects of RT in the short-term may be less clear, with several recent smaller studies reporting inconsistent findings related to the relationship between RT exposure and cardiac function (7-10). Moreover, in the modern treatment era, the cardiac side effects of RT may be decreased due to improvements in radiation planning and delivery that reduce heart dose (11).

To clarify the early cardiac effects of contemporary RT in breast cancer patients, we performed detailed echocardiographic phenotyping prior to, immediately following, and 5-9 months after thoracic RT exposure in a prospective longitudinal cohort study, the $\mathrm{XXXX}(\mathrm{XXXX)}$ study. Specifically, we sought to determine the changes in cardiac function that occur acutely and subacutely following RT exposure in breast cancer patients and the associations with MHD. We hypothesized that thoracic RT results in a dose-dependent worsening of cardiovascular function as defined by echocardiographic-derived measures of left ventricular ejection fraction (LVEF), longitudinal and circumferential strain, and diastolic function.

\section{METHODS}

\section{Study Population}


We prospectively enrolled breast cancer patients newly initiating photon or proton

2 thoracic RT from the Department of Radiation Oncology at the XXXX from June 2015 to

3 January 2018. Inclusion criteria were age greater than 18 years old; left or right-sided breast cancer newly initiating fractionated whole-breast/chest wall RT with an expected cardiac dose or regional nodal photon or proton $\mathrm{RT}$; or right-sided breast cancer with internal mammary nodal RT who were newly starting fractionated photon or proton RT were also included with an expected cardiac dose. The expected cardiac dose was determined by the treating radiation oncologist, with the goal of enrolling patients who would have any expected more than minimal risk heart dose from whole breast $\mathrm{RT}$ on the left, or regional internal mammary node treatment on either side. Patients were included regardless of exposure to adjuvant chemotherapy, neoadjuvant chemotherapy, and/or targeted therapy. Also, patients with a history of prior contralateral breast radiation without cardiac involvement were eligible for recruitment. Patients were excluded if they had prior RT to the thoracic region that would result in overlap of RT fields; life expectancy less than 12 months; lack of echocardiographic imaging due to lack of baseline echocardiograms or poor acoustic windows; or inability to comprehend English. The study was approved by the XXXX Institutional Review Board.

\section{Study procedures}

Detailed clinical data, demographics, cardiovascular, and oncologic history were collected using standardized patient and physician questionnaires and verified through medical records review. We obtained these data at three time points: within 4 weeks before RT (T0), within 3 days prior to the end of RT to 6 weeks after the end of RT (T1), and 5-9 months after RT completion (T2) (Figure 1). In our analysis, we defined acute changes as those from T0 to $\mathrm{T} 1$ and subacute changes as those from $\mathrm{T} 1$ to $\mathrm{T} 2$. 


\section{Cardiac radiation exposure}

Study patients underwent planning computed tomography (CT) or positron emission tomography (PET/CT) simulation with appropriate positioning and immobilization with or without deep inspiratory breath-hold (DIBH) technique as per institutional standard of care. 4D CT data were used to ascertain if the patient would benefit from DIBH by evaluating the relative heart position compared to the breast clinical target volume of the maximum inhaled CT phase and compared to maximum exhaled CT phase. DIBH was used when possible. Normal anatomic structures were contoured by dosimetrists. Radiation target volumes and margins were contoured by the treating radiation oncologist using the standardized contouring protocol based on Radiotherapy Comparative Effectiveness Consortium (RADCOMP) atlas (12-14). Radiation treatment planning using 3-D conformal, intensity-modulated radiation therapy (IMRT) or proton therapy was performed by dosimetrists/medical physicists using institutional dose constraints, which are based on modern Radiation Therapy Oncology Group (RTOG) dose guidelines (15). After completion of the radiation plan, dose parameters to organs-at-risk were extracted from dose-volume histograms, including MHD, and the percent volume of heart receiving incremental radiation doses (e.g., V5 Gy, V20 Gy). All dosimetry calculations were performed using Eclipse Treatment Planning System v 15.6 (Varian Medical Systems, Palo Alto, CA, USA).

\section{Quantitative Echocardiography}

Two-dimensional and Doppler echocardiography were performed on Vivid E9 or E95 machines (GE Healthcare, Milwaukee, WI) according to a standard research protocol. Images were quantified in a blinded manner by sonographers at the XXXX Center for Quantitative Echocardiography using the TomTec® Imaging Systems platform (Unterschleissheim, Germany). LVEF was calculated using biplane Simpson's method of discs using left ventricular end-diastolic and end-systolic volumes in four and two chambers views (16). Longitudinal strain 
1 and circumferential strain were quantified on images, digitally archived at 60-80 frames per

2 second, using vendor-independent software (Cardiac Performance Analysis, TomTec Imaging

3 Systems). Diastolic dysfunction was assessed by measurement of transmitral flow parameters

4 (early transmitral flow $(E)$, late transmitral flow $(A)$, deceleration time), tissue Doppler indices of mitral annular velocity (septal and lateral e'), left atrial volume indexed to body surface area (LAVI) and tricuspid regurgitation (TR) velocity. Average E/e' is a measure of diastolic function, which represents the early transmitral inflow divided by the average of the septal and lateral tissue Doppler indices e'. This ratio is often used as a measure of left-sided cardiac filling pressures. Estimates of the intraobserver coefficient of variation were $4.4 \%$ for LVEF, $10.9 \%$ for longitudinal strain, and $9.4 \%$ for circumferential strain $(17,18)$. The intraobserver coefficients of variation for mitral inflow and tissue Doppler velocities were $2.3 \%$ to $5.4 \%$ (19). All quantitation was performed by observers blinded to patient characteristics and timing of echocardiograms.

\section{Statistical Analysis}

Standard descriptive statistics were used to characterize the study population at baseline using proportions for categorical variables and mean (standard deviation) and median (interquartile range (IQR)) for normally and non-normally distributed continuous variables, respectively. Differences in echocardiographic measures at T0 and T1 (acute), as well as T0 and T2 (subacute), were tested with the Wilcoxon signed-rank test. Differences in echocardiographic measures across all three time points were tested with the Kruskal-Wallis test. We evaluated the following echocardiographic outcomes: LVEF, longitudinal strain, circumferential strain, and E/e'.

We then assessed the multivariable associations between changes in each echocardiographic measure and MHD in the acute (T0 to T1) and subacute (T0 to T2) time period. We used generalized estimating equations (GEE) with an exchangeable working 
1 correlation structure for the longitudinal observations of echocardiographic measures. GEE was

2 chosen as we wanted to quantify the population-level associations between change of cardiac

3 function and MHD, although sensitivity analyses using linear mixed models was also performed.

Days from RT was calculated as the time from the first day of initiating RT therapy as a continuous variable. For each model, confounders were selected based on a combination of statistical evidence with clinical and biological judgment. We a priori hypothesized that the effect of MHD on cardiac function was not constant over time, and thus modeled the changes from T0 to T1 and T2 separately. We also hypothesized that the association between MHD and echocardiographic-derived measures of cardiac function would differ according to systemic cancer therapy exposure, and explored the interaction between cancer therapy (anthracyclines, trastuzumab) and MHD at baseline $(20,21)$.

We developed three sequential models: Model 1 included the longitudinal assessments of our echocardiographic variable of interest as the outcome, MHD, time from radiation treatment, and a time by exposure interaction with MHD as our primary variable of interest; Model 2 included the variables in Model 1 in addition to baseline (T0) echocardiographic parameter, age, race, pre-existing cardiovascular disease, anthracycline and/or trastuzumab exposure. Pre-existing cardiovascular disease was defined as a history of hypertension, hyperlipidemia, diabetes, coronary artery disease including a history of angina or previous myocardial infarction, arrhythmias, heart failure, known history of reduced ejection fraction, or cardiac surgery; Model 3, the fully adjusted model, included variables in Model 2 and an interaction term with anthracycline or trastuzumab exposure and MHD. All tests were two-sided; the type I error rate was set at 0.05. Statistical analyses were performed using STATA V.15.1 (StataCorp, College Station, TX) (22). Graphics were generated using the ggplot2 package in R version 3.6.1 (R Foundation for Statistical Computing, Vienna, Austria) (23). Assuming a standard deviation of 3-4\%, a sample size of 86 with two repeated follow-up measurements was 
estimated to provide $>80 \%$ power to detect an absolute change relative to baseline as low as $1.0 \%$ for LVEF, longitudinal strain, and circumferential strain at a two-sided significance level of $5 \%$.

\section{RESULTS}

\section{Study population}

Eighty-six female patients were enrolled (Table 1). The mean age at enrollment was 53 \pm 11.4 years; the median was 54 years [IQR 44, 62 years]. At baseline, 68 (79.1\%) had primary left-sided breast cancer, $10(11.6 \%)$ had primary right-sided breast cancer, $3(3.5 \%)$ had recurrent breast cancer, and 5 (5.8\%) had bilateral breast cancer. Overall, 9 patients $(10.5 \%)$ had stage 0, $18(20.9 \%)$ had stage I, $32(37.2 \%)$ had stage II, and $26(30.2 \%)$ had stage III breast cancer (24). There were 18 patients (20.9\%) who had no systemic cancer therapy as part of their current treatment regimen; however, 48 (55.8\%) had only anthracycline exposure, 20 (23.3\%) had trastuzumab exposure, and 11 (12.8\%) had both anthracycline and trastuzumab exposure.

Of the 86 patients with analyzable echocardiograms at baseline (T0), 79 patients were evaluated at T1, and 83 evaluated at T2. The median time from RT initiation to T1 was 56 days [IQR 43, 72 days], and T2 was 223 days [IQR 196, 259 days]. Seventeen additional studies from sixteen individual patients were performed for clinical indications outside the prespecified timepoints and included in the analyses. These additional studies were primarily performed for standard monitoring indications during trastuzumab therapy. Two studies occurred between T0 and T1 (17 and 29 days after RT) and 15 studies occurred between T1 and T2 visits (median 148 days after RT, [IQR 121, 168 days]).

\section{Radiation therapy}


Across the entire cohort, the median MHD was low, at 139 cGy [IQR 99, 249 cGy] with a range of 2-789 cGy. In patients with primary left-sided breast cancer, the median MHD was 142 cGy [IQR 104, 264 cGy]; in primary right-sided breast cancer, the MHD was 86 cGy [IQR 34, 129 cGy]; in bilateral breast cancer, the MHD was 210 cGy [IQR 181, 302 cGy]; and in recurrent breast cancer, the MHD was 115 cGy [IQR 103, 288 cGy]. The primary RT technique was 3D conformal (tangential fields) in 61 patients $(70.9 \%), 12$ patients $(14.0 \%)$ had IMRT (tangential fields), 9 patients (10.5\%) had scanning proton radiation, and 4 patients $(4.7 \%)$ had passive scattering proton radiation using either two or three non-tangential fields. The median time between RT initiation and completion was 40 days (IQR 30, 44 days]. Thirty-seven patients had motion management techniques used during RT therapy with a median MHD was 144 cGy [IQR 115, 327 cGy] compared to 120 cGy [IQR 99, 224 cGy] in those without motion management techniques. A majority (28 patients) utilized DIBH with a median MHD 205 cGy [IQR 119, 360 cGy], 8 patients were placed in prone positioning with MHD 109 cGy [IQR 91, 128 cGy], and 1 subject had abdominal compression with MHD of 186 cGy. Sixty-two out of 86 patients $(72.0 \%)$ had boost radiation with 6 patients $(7.0 \%)$ having proton therapy, 40 patients (46.5\%) having photon therapy, and 16 patients (18.6\%) having electron therapy. The median total RT dose was 5256 cGy [IQR 5040, 6000 cGy] with a median dose of initial fields of 5000 cGy [IQR 4256, 5040 cGy]. A median cone down dose of 1000 cGy [IQR 1000, 1000 cGy] was used when clinically indicated.

Changes in systolic function pre-RT (T0), immediately after RT (T1), and 5-9 months after RT (T2)

Changes in echocardiographic markers over time from baseline are presented in Figure 2 and Table 2. First, we examined longitudinal patterns in echocardiographic measures over days from RT exposure (Figure 2). At T0, the median LVEF was 53.0\% [IQR 50.0\%, 55.8\%], 
1 likely reflective of the effects of anthracyclines and/or trastuzumab therapy administered prior to

2 RT. At T0, 21 patients of 86 had a quantitated LVEF less than $50 \%$ at baseline, prior to RT. The median LVEF in this subgroup was only mildly decreased at $48.0 \%$ [IQR $44.9 \%, 48.9 \%$ ]. At T1, the median LVEF was 51.5\% [IQR 48.0\%, 54.1\%], and at T2 was 52.4\% [IQR 49.9\%, 55.3\%] (Table 2). There was a modest decrease in the absolute LVEF from T0 to T1 of $-1.8 \%(95 \% \mathrm{Cl}$ $2.8 \%,-0.7 \% ; p=0.01)$. However, there was no significant difference in the LVEF from T0 to T2 (Figure 2A, Table 2).

At T0, the median longitudinal strain was $-15.6 \%$ [IQR $-18.1 \%,-13.1 \%$, again likely reflective of the cardiotoxic effects of anthracyclines and/or trastuzumab therapy. At T1, the median longitudinal strain was $-15.6 \%$ [IQR $-18.9 \%,-13.7 \%]$ and at T2 longitudinal strain was $16.8 \%$ [IQR $-20.2 \%,-14.9 \%]$. There were no differences in longitudinal strain between T0 and $\mathrm{T} 1$ with a mean change in longitudinal strain of $-0.5 \%(95 \% \mathrm{Cl}-1.4,0.4 ; \mathrm{p}=0.33)$. However, between $\mathrm{T} 1$ and $\mathrm{T} 2$, there was a very modest improvement in longitudinal strain that was of borderline statistical significance (mean change $-0.9 \% ; 95 \% \mathrm{Cl}-1.7 \%,-0.1 \% ; p=0.06$ ). In comparing T0 and T2, longitudinal strain was also improved at T2 with a mean change in longitudinal strain of $-1.8 \%(95 \% \mathrm{Cl}-2.6,-0.9 ; \mathrm{p}=0.003)$ (Figure $2 \mathrm{~B})$. The median circumferential strain changes over time are detailed in Table 2; there were no differences in circumferential strain between T0 and T1 or T2 (Figure 2C).

\section{Associations between echocardiographic measures of systolic function and mean heart dose}

We next determined the associations between the absolute change in LVEF and MHD accounting for the time from RT exposure. Here, the reported beta coefficients represented the change in each echocardiographic measure per 30-day interval for every 100 cGy increase of 
1 RT exposure (Table 3). Between T0 and T1, there were no significant associations in our

2 unadjusted and minimally adjusted models in GEE analysis. Accounting for confounders and the

3 interaction between MHD and anthracyclines and/or trastuzumab exposure in Model 3, there was a borderline significant $0.22 \%$ decrease in LVEF $(95 \% \mathrm{Cl}-0.44 \%, 0.01 \% ; p=0.06)$ per 30 day interval for every 100 cGy increase of RT exposure from T0 to T1. This translates to an annualized decrease in LVEF of $2.6 \%(95 \% \mathrm{Cl}-5.3 \%, 0.1 \%)$ at T1 compared to T0 for every 100 cGy of RT exposure. There was no association between MHD and the rate of change in LVEF from T0 to T2. Additionally, we present the regression coefficients of the explored interaction term between MHD (per 100 cGy) and anthracycline and/or trastuzumab exposure in the Supplement Table S5. In these exploratory analyses, trastuzumab was observed to be an effect modifier of the cross-sectional associations between MHD and cardiac function.

For longitudinal strain, we found no significant associations between MHD and the rate of change in strain in the acute unadjusted and minimally adjusted models from T0 to T1. In the acute fully adjusted model (Model 3) there was a modest worsening in longitudinal strain, of borderline statistical significance, at 30 days for each 100 cGy increase in MHD on the order of $0.19 \%(95 \% \mathrm{Cl}-0.01 \%, 0.39 \% ; \mathrm{p}=0.06)$ (Table 3$)$ which translates into an annualized worsening in longitudinal strain of $2.3 \%(95 \% \mathrm{Cl}-0.1 \%, 4.7 \%)$. Between $\mathrm{T} 0$ and $\mathrm{T} 2$, there were no subacute associations between longitudinal strain and MHD. Furthermore, there were no associations with MHD and rate of change in circumferential strain from T0 to T1 or from T0 to T2.

In additional sensitivity analyses evaluating the association between V5, V10, and V20 and longitudinal and circumferential strain, our findings were similar (Supplemental Tables S13). We performed additional analyses evaluating the association between acute changes in LVEF across a spectrum of heart dose-volume parameters and found similar modest 
associations (Supplemental Table S4). Finally, sensitivity analyses using fully adjusted linear mixed models showed similar associations as in our GEE analyses (Supplemental Table 6).

\section{Changes in diastolic function pre-RT, immediately after RT and 5-9 months after RT}

There were no overall differences in E/e' throughout follow-up (Figure 2D, Table 2). The median E/e' at T0 was 7.9 (IQR 6.6, 10.3); at T1 was 8.3 (IQR 6.7, 9.9); and at T2 was 8.1 (IQR $6.8,10.8)$. There was a decrease in left atrial volume indexed to body surface area (LAVI) from $22.9 \mathrm{ml} / \mathrm{m} 2($ IQR $20.0,27.5)$ at T0 to $20.5 \mathrm{ml} / \mathrm{m} 2($ IQR $16.6,24.3)$ at T2 $(\mathrm{p}=0.006)$. There were no changes in E/e', E/A, or other diastolic function parameters at the three time points (Table 2).

\section{Associations between echocardiographic measures of diastolic function and mean heart} dose

In our multivariable GEE analysis (Table 3), there were no acute associations between E/e' and MHD between T0 and T1. In evaluating the unadjusted associations between E/e' and MHD from T0 to T2, there was a very small increase in average E/e' of $0.07(95 \% \mathrm{Cl} 0.001$, 0.13; $p=0.05$ ) per 30-day interval for every 100 cGy of RT exposure. In multivariable models, the effect size was similar, although this association was not statistically significant. We again performed additional sensitivity analyses evaluating the association between $\mathrm{V} 5, \mathrm{~V} 10$, and $\mathrm{V} 20$ and diastolic dysfunction, and our findings were similar (Supplemental Table S1-3).

\section{DISCUSSION}

In this prospective longitudinal cohort study of 86 breast cancer patients, we evaluated the changes in cardiac function prior to, immediately after, and within nine months after RT. 
1 With modern radiation planning techniques, the MHD is low. We observed three main findings:

2 1) there was a slight worsening in LVEF acutely after RT that recovered over time; 2 ) there was

3 no change in longitudinal strain, circumferential strain, or E/e' immediately after RT; 3) the associations between MHD and core-lab quantified measures of systolic function (LVEF, longitudinal strain) were modest, demonstrating only a slight decrease in cardiac function per 100 cGy increase in MHD. Our results suggest that the short-term, adverse effects of RT in breast cancer on subclinical measures of cardiovascular function are overall very modest.

We found a small decrease in LVEF in the acute time period from pre-RT to immediately post RT, which recovered in the subacute period 5-9 months post-RT, and an association between MHD exposure and change in LVEF that was of borderline statistical significance. Other smaller, retrospective studies $(\mathrm{N}=47)$ have also reported significant changes in measures of LVEF but did not determine an association with MHD (25). We hypothesize that the smaller sample size of these other studies and the limited power, as well as the small effect sizes, may explain the disparate findings.

Consistent with our findings above, we also determined a weak association between longitudinal strain and MHD in our multivariable analyses. We also observed an abnormal preRT longitudinal strain that we hypothesize is reflective of the effects of anthracycline chemotherapy (18). Prior studies evaluating longitudinal strain have primarily focused on chemotherapy or targeted therapy naïve patients receiving a greater MHD. For example, Lo et al. studied left-sided breast cancer patients treated with breast-conserving surgery who were chemotherapy naïve and only treated with adjuvant RT with a mean MHD of $250 \pm 130$ cGy and no nodal RT (26). Over six-weeks of follow-up, there was a reduction in global and segmental systolic strain parameters compared with baseline assessments with the most significant decrement in the left ventricle (LV) apical segments. Similar findings were observed by Tuohinen et al. (27) and others in chemotherapy naïve breast cancer patients treated with 
$1 \mathrm{RT}(28)$. Altogether, these data suggest that in the acute period following RT exposure, there are

2 small changes in LVEF and longitudinal strain that are likely dose dependent. The clinical significance of these changes remains to be determined, and longer-term follow-up is needed.

We did not find an association between MHD and the change in circumferential strain in our analysis, consistent with previously published studies $(7,25)$. Circumferential strain tends to be reflective of a more advanced stage of cardiac injury and remodeling (17), also consistent with these findings.

We also did not identify changes in indices of diastolic function. Our longitudinal data analysis did demonstrate an association between MHD and E/e' in the subacute period in our minimally adjusted models, which was no longer significant after multivariable adjustment. The literature regarding changes in diastolic dysfunction after RT exposure has been mixed or not reported $(8,9,25,29,30)$. The pathophysiology of $\mathrm{RT}$ leading to diastolic dysfunction remains to be fully elucidated, as does the question of whether small changes in diastolic function contribute to clinical disease (31).

The cardiovascular concerns of complications from modern-day RT may be less significant than the historical literature, particularly with the current advances in RT delivery and low MHD. We attribute the results of our study primarily to the successes in minimizing cardiac exposure in thoracic RT with cardiac contouring and with the increased use of DIBH techniques and some patients receiving proton therapy. Of note, the MHD in our longitudinal study was significantly lower than the previously reported series $(7,9,10,26,32-35)$.

\section{Strengths and Limitations}

The strengths of this study included the large sample size compared with the previous series $(7,25,26)$, the detailed phenotyping of our patients' treatment exposures, rigorously 
1 quantitated echocardiographic outcome measures, as well as the use of a prospective

2 longitudinal study design. To date, studies assessing acute radiation cardiotoxicity have had

3 small patient numbers without the use of contemporary RT techniques and anticancer regimens

$4 \quad(7,26)$. Our study design and patient enrollment more accurately reflect current chemotherapy

5 and RT practices for breast cancer more so than previously reported in the literature (7-

$610,26,32,33)$. The $X X X X$ cohort had a high retention rate with minimal missing data $(<5 \%)$.

Limitations included a relatively short median follow-up time of 7.3 months after RT, but our focus is on early changes immediately post RT. Although larger than previously reported studies, our sample size was still small, limiting statistical power and the ability to perform a detailed comparison of proton and photon RT. We included all patients regardless of chemotherapy or targeted therapy history to enhance the generalizability of our findings. However, we acknowledge that we cannot fully differentiate the effects of RT from the effects of cardiotoxic systemic cancer therapy, given our relatively small sample size. We adjusted for anthracycline and trastuzumab exposure in our multivariable models but acknowledge that this may not fully account for the complex interactions between systemic cardiotoxic therapy and $\mathrm{RT}$, particularly given toxicity is known to be dose-dependent. We did not include cardiac subsite-specific analysis such as left anterior descending artery exposure, left ventricle, and right ventricle, given the limited MHD exposure, but this should be considered in future studies. Our cohort was enrolled at a tertiary center with state-of-the-art imaging-guided RT delivery techniques, limiting generalizability to the broader population of breast cancer patients.

\section{CONCLUSIONS}

This study provides insight into several knowledge gaps in breast cancer patients undergoing systemic cancer therapy and RT. In the era of contemporary thoracic RT for breast cancer, the degree of subclinical cardiac injury that occurs acutely and subacutely during RT 
1 exposure is minimal and likely related to the low MHD. Longer-term follow-up studies are

2 needed to understand if these subclinical changes are clinically relevant and if they contribute to 3 late clinical cardiovascular disease. 


\section{References}

1. Ebctcg, McGale $P$, Taylor $C$, et al. Effect of radiotherapy after mastectomy and axillary surgery on 10-year recurrence and 20-year breast cancer mortality: Meta-analysis of individual patient data for 8135 women in 22 randomised trials. Lancet (London, England) 2014;383:2127-2135.

2. Bouillon K, Haddy N, Delaloge S, et al. Long-term cardiovascular mortality after radiotherapy for breast cancer. Journal of the American College of Cardiology 2011;57:445-452.

3. Darby SC, Ewertz M, McGale $P$, et al. Risk of ischemic heart disease in women after radiotherapy for breast cancer. N Engl J Med 2013;368:987-98.

4. Saiki H, Petersen IA, Scott CG, et al. Risk of heart failure with preserved ejection fraction in older women after contemporary radiotherapy for breast cancer. Circulation 2017;135:1388-1396.

5. Heidenreich PA, Kapoor JR. Radiation induced heart disease. Heart 2009;95:252-258.

6. Bovelli D, Plataniotis G, Roila F, et al. Cardiotoxicity of chemotherapeutic agents and radiotherapy-related heart disease: Esmo clinical practice guidelines. Ann Oncol 2010;21 Suppl 5:v277-82.

7. Lo Q, Hee L, Batumalai V, et al. Subclinical cardiac dysfunction detected by strain imaging during breast irradiation with persistent changes 6 weeks after treatment. Int $J$ Radiat Oncol Biol Phys 2015;92:268-76.

8. Sritharan HP, Delaney GP, Lo Q, et al. Evaluation of traditional and novel echocardiographic methods of cardiac diastolic dysfunction post radiotherapy in breast cancer. Int J Cardiol 2017;243:204-208.

9. Erven K, Florian A, Slagmolen $\mathrm{P}$, et al. Subclinical cardiotoxicity detected by strain rate imaging up to 14 months after breast radiation therapy. Int $J$ Radiat Oncol Biol Phys 2013;85:1172-8.

10. Erven $\mathrm{K}$, Jurcut $\mathrm{R}$, Weltens $\mathrm{C}$, et al. Acute radiation effects on cardiac function detected by strain rate imaging in breast cancer patients. Int J Radiat Oncol Biol Phys 2011;79:144451.

11. Killander F, Wieslander E, Karlsson $P$, et al. No increased cardiac mortality or morbidity of radiation therapy in breast cancer patients after breast-conserving surgery: 20-year follow-up of the randomized swebcgrt trial. Int J Radiat Oncol Biol Phys 2020;107:701709.

12. Atlas RB. In: Editor, editor^editors. Book.

13. Feng M, Moran JM, Koelling $T$, et al. Development and validation of a heart atlas to study cardiac exposure to radiation following treatment for breast cancer. International Journal of Radiation Oncology ${ }^{*}$ Biology ${ }^{*}$ Physics 2011;79:10-18.

14. Duane F, Aznar MC, Bartlett F, et al. A cardiac contouring atlas for radiotherapy. Radiotherapy and Oncology 2017;122:416-422.

15. Mamounas E. A randomized phase iii clinical trial evaluating post-mastectomy chestwall and regional nodal xrt and post-lumpectomy regional nodal xrt in patients with positive axillary nodes before neoadjuvant chemotherapy who convert to pathologically negative axillary nodes after neoadjuvant chemotherapy. In: Editor, editor ${ }^{\wedge}$ editors. Book A randomized phase iii clinical trial evaluating post-mastectomy chestwall and regional nodal xrt and post-lumpectomy regional nodal xrt in patients with positive axillary nodes before neoadjuvant chemotherapy who convert to pathologically negative axillary nodes after neoadjuvant chemotherapy; 2013. pp. NSABP B-51/RTOG 1304.

16. Lang RM, Badano LP, Mor-Avi V, et al. Recommendations for cardiac chamber quantification by echocardiography in adults: An update from the american society of 
echocardiography and the european association of cardiovascular imaging. J Am Soc Echocardiogr 2015;28:1-39 e14.

17. Narayan HK, French B, Khan AM, et al. Noninvasive measures of ventricular-arterial coupling and circumferential strain predict cancer therapeutics-related cardiac dysfunction. JACC Cardiovasc Imaging 2016;9:1131-1141.

18. Demissei BG, Freedman G, Feigenberg SJ, et al. Early changes in cardiovascular biomarkers with contemporary thoracic radiation therapy for breast cancer, lung cancer, and lymphoma. Int J Radiat Oncol Biol Phys 2019;103:851-860.

19. Upshaw JN, Finkelman B, Hubbard RA, et al. Comprehensive assessment of changes in left ventricular diastolic function with contemporary breast cancer therapy. JACC CardiovasC Imaging 2020;13:198-210.

20. Armenian SH, Lacchetti C, Barac A, et al. Prevention and monitoring of cardiac dysfunction in survivors of adult cancers: American society of clinical oncology clinical practice guideline. J Clin Oncol 2017;35:893-911.

21. van Nimwegen FA, Ntentas G, Darby SC, et al. Risk of heart failure in survivors of hodgkin lymphoma: Effects of cardiac exposure to radiation and anthracyclines. Blood 2017;129:2257-2265.

22. StataCorp. Stata statistical software: Release 15. In: Editor, editor^editors. Book Stata statistical software: Release 15. College Station, TX: StataCorp LLC; 2017.

23. Team RC. R: A language and environment for statistical computing. In: Editor, editor^editors. Book R: A language and environment for statistical computing. Vienna, Austria: R Foundation for Statistical Computing; 2019.

24. Amin MB, Edge SB. Ajcc cancer staging manual: Springer; 2017.

25. Yu AF, Ho AY, Braunstein LZ, et al. Assessment of early radiation-induced changes in left ventricular function by myocardial strain imaging after breast radiation therapy. Journal of the American Society of Echocardiography 2019;32:521-528.

26. Lo Q, Hee L, Batumalai V, et al. Strain imaging detects dose-dependent segmental cardiac dysfunction in the acute phase after breast irradiation. Int $J$ Radiat Oncol Biol Phys 2017;99:182-190.

27. Tuohinen SS, Skyttä T, Poutanen T, et al. Radiotherapy-induced global and regional differences in early-stage left-sided versus right-sided breast cancer patients: Speckle tracking echocardiography study. The international journal of cardiovascular imaging 2017;33:463-472.

28. Murphy AC, Lancefield TF, Chao M, et al. Assessment of cardiac function in chemotherapy naive women with breast cancer undergoing contemporary radiation therapy. JACC: CardioOncology 2020;2:509-510.

29. Heidenreich PA, Hancock SL, Vagelos $\mathrm{RH}$, et al. Diastolic dysfunction after mediastinal irradiation. American Heart Journal 2005;150:977-982.

30. Cao L, Cai G, Chang C, et al. Diastolic dysfunction occurs early in her2-positive breast cancer patients treated concurrently with radiation therapy and trastuzumab. Oncologist 2015;20:605-14.

31. Sardaro A, Petruzzelli MF, D'Errico MP, et al. Radiation-induced cardiac damage in early left breast cancer patients: Risk factors, biological mechanisms, radiobiology, and dosimetric constraints. Radiotherapy and oncology : journal of the European Society for Therapeutic Radiology and Oncology 2012;103:133-142.

32. van den Bogaard VAB, van Luijk $P$, Hummel YM, et al. Cardiac function after radiation therapy for breast cancer. Int J Radiat Oncol Biol Phys 2019;104:392-400.

33. Trivedi SJ, Choudhary P, Lo Q, et al. Persistent reduction in global longitudinal strain in the longer term after radiation therapy in patients with breast cancer. Radiotherapy and oncology : journal of the European Society for Therapeutic Radiology and Oncology 2019;132:148-154. 
34. Freedman RA, Fedewa SA, Punglia RS, et al. Factors associated with radiation therapy incompletion for patients with early-stage breast cancer. Breast Cancer Res Treat 2016;155:187-99.

35. Beck RE, Kim L, Yue NJ, et al. Treatment techniques to reduce cardiac irradiation for breast cancer patients treated with breast-conserving surgery and radiation therapy: A review. Front Oncol 2014;4:327. 


\section{Figures}

Figure 1 - XXXX (XXXX) study design. Patient clinical data, demographics, cardiovascular, and oncologic history were assessed at three time points: within 4 weeks before RT (T0); within 3 days prior to the end of RT to 6 weeks after the end of RT (T1), and 5-9 months after RT completion (T2). RT was typically an 8-week course with a median of 40 days of treatment, RT = Radiation Therapy

Figure 2 - Changes in echocardiographic measures (LVEF, longitudinal strain, circumferential strain, and E/e') from baseline values after RT therapy. Gray lines represent the trajectory of each echo variable plotted for each individual subject. Blue lines indicate summary Loess smoothing splines which represent the mean change in each echocardiographic measure with point-wise confidence bands. Changes for each echocardiographic variable are represented in A) LVEF (\%), B) Longitudinal strain (\%), C) Circumferential strain (\%), and D) E/e'. For longitudinal and circumferential strain, which are negative values, a decrease from baseline represents an improvement in strain, for example, from -15.6 to $\%-16.8 \%$. E/e' = diastolic function index; LVEF = left ventricular ejection fraction; RT = Radiation Therapy 


\begin{tabular}{|c|c|}
\hline & 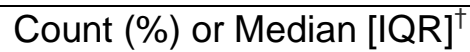 \\
\hline Age at diagnosis (years) & $54[44,62]$ \\
\hline \multicolumn{2}{|l|}{ Race } \\
\hline Caucasian & $62(72.1)$ \\
\hline Black or African American & $21(24.4)$ \\
\hline Asian/Pacific Islander & $3(3.5)$ \\
\hline Baseline BMI, kg/m2 & $28.4[23.6,32.8]$ \\
\hline Current or past smoking & $36(41.9)$ \\
\hline \multicolumn{2}{|l|}{ Cardiovascular history } \\
\hline Hypertension & $29(33.7)$ \\
\hline Diabetes Mellitus & $10(11.6)$ \\
\hline Hyperlipidemia & $23(26.7)$ \\
\hline Coronary artery disease (CAD) & $4(4.7)$ \\
\hline History of heart failure (HF) & $5(5.8)$ \\
\hline Pre-existing cardiovascular disease or risk factors ${ }^{\star *}$ & $45(52.3)$ \\
\hline ACEI/ARB or beta-blocker use at baseline & $29(33.7)$ \\
\hline \multicolumn{2}{|l|}{ Breast Cancer Site } \\
\hline Primary left & $68(79.1)$ \\
\hline Primary right & $10(11.6)$ \\
\hline Recurrent & $3(3.5)$ \\
\hline Bilateral & $5(5.8)$ \\
\hline \multicolumn{2}{|l|}{ AJCC Breast Cancer Stage } \\
\hline Stage 0 & $9(10.5)$ \\
\hline Stage 1 & $18(20.9)$ \\
\hline Stage 2 & $32(37.2)$ \\
\hline Stage 3 & $26(30.2)$ \\
\hline \multicolumn{2}{|l|}{ Anthracycline (AC) or trastuzumab $(T)$ exposure } \\
\hline Neither AC nor T & $18(20.9)$ \\
\hline Anthracycline & $48(55.8)$ \\
\hline Trastuzumab & $20(23.3)$ \\
\hline Both $A C$ and $T$ & $11(12.8)$ \\
\hline \multicolumn{2}{|l|}{ Primary radiation technique } \\
\hline Protons (passive scattering) & $4(4.7)$ \\
\hline Protons (scanning) & $9(10.5)$ \\
\hline 3D Conformal & $61(70.9)$ \\
\hline IMRT & $12(14.0)$ \\
\hline Mean Heart Dose (MHD) (cGy) & $139[99,249]$ \\
\hline V5 cGy Heart Dose (\%) & $2.8[1.3,8.5]$ \\
\hline V10 cGy Heart Dose (\%) & $1.1[0.2,3.9]$ \\
\hline V20 cGy Heart Dose (\%) & $0.35[0.01,1.6]$ \\
\hline LVEF baseline (\%) & $53.0[50.0,55.8]$ \\
\hline Longitudinal strain baseline (\%) & $-15.6[-18.1,-13.1]$ \\
\hline Circumferential Strain baseline (\%) & $-23.5[-27.6,-19.7]$ \\
\hline E/e' average baseline & $7.9[6.6,10.3]$ \\
\hline
\end{tabular}

Abbreviations:3D = 3-dimensional; $\mathrm{AC}$ = anthracycline; $\mathrm{ACEi}$ = angiotensin-converting enzyme inhibitor; AJCC = American Joint Committee on Cancer; $\mathrm{ARB}=$ angiotensin receptor blocker; $\mathrm{BMI}=$ body mass index; $C A D$ = coronary artery disease; $E / e^{\prime}=$ diastolic function index; $H F$ = heart failure 
IMRT = intensity-modulated radiation therapy; MHD = mean heart dose; $\mathrm{RT}=$ radiation therapy; $\mathrm{T}=$ trastuzumab

† Categorical variables were summarized with count (\%); all continuous variables were summarized with the median [interquartile range]

** Pre-existing cardiovascular disease was defined as the diagnosis or history of hypertension, hyperlipidemia, diabetes, coronary artery disease including a history of angina or previous myocardial infarction, arrhythmias, heart failure, or a known history of reduced ejection fraction, or cardiac surgery. 
Table 2 - Echocardiographic Parameters Pre-RT (T0), Immediately Post-RT (T1) and 5-9 months after RT completion (T2)

\begin{tabular}{|c|c|c|c|c|}
\hline $\begin{array}{c}\text { Echocardiographic measure, } \\
\text { median [IQR] }\end{array}$ & $\begin{array}{c}\text { T0 } \\
\text { Pre-RT } \\
\mathrm{N}=86\end{array}$ & $\begin{array}{c}\mathrm{T} 1 \\
\text { Immediately post- } \\
\mathrm{RT} \\
\mathrm{N}=79\end{array}$ & $\begin{array}{c}\mathrm{T} 2 \\
\text { 5-9 Months post-RT } \\
N=83\end{array}$ & $\begin{array}{c}p- \\
\text { value } \\
\dagger\end{array}$ \\
\hline LVEF (\%) & $53.0[50.0,55.8]$ & $51.5[48.0,54.1] \ddagger$ & $52.4[49.9,55.3]$ & 0.04 \\
\hline Longitudinal strain (\%) & $-15.6[-18.1,-13.1]$ & $-15.6[-18.9,-13.7]$ & $-16.8[-20.2,-14.9]^{\star *}$ & 0.01 \\
\hline Circumferential Strain (\%) & $-23.5[-27.6,-19.6]$ & $-24.1[-29.0,-20.9]$ & $-24.5[-30.3,-20.4]$ & 0.30 \\
\hline Left Atrial Volume Index $\mathrm{mL} / \mathrm{m} 2$ & $22.9[20.0,27.5]$ & $22.0[18.8,25.0]$ & $20.5[16.6,24.3]^{\star \star}$ & 0.02 \\
\hline E wave $(\mathrm{cm} / \mathrm{s})$ & $74.0[62.0,90.0]$ & $78.5[64.0,91.0]$ & $74.0[62.0,83.0]$ & 0.52 \\
\hline A wave $(\mathrm{cm} / \mathrm{s})$ & $68.0[58.0,82.5]$ & $70.5[58.0,86.0]$ & $71.0[57.0,83.0]$ & 0.96 \\
\hline LV Lateral Velocity e wave $(\mathrm{cm} / \mathrm{s})$ & $10.0[8.0,12.0]$ & $10.0[8.0,12.0]$ & $10.0[8.0,12.0]$ & 0.36 \\
\hline LV Lateral Velocity a wave $(\mathrm{cm} / \mathrm{s})$ & $10.0[8.0,11.0]$ & $9.0[8.0,10.0]$ & $9.0[7.0,10.0]$ & 0.21 \\
\hline LV Septal Velocity e wave (cm/s) & $7.0[6.0,10.0]$ & $8.0[6.0,10.0]$ & $7.0[6.0,10.0]$ & 0.29 \\
\hline LV Septal Velocity a wave $(\mathrm{cm} / \mathrm{s})$ & $9.0[7.0,10.0]$ & $8.5[7.0,10.0]$ & $8.0[8.0,10.0]$ & 0.72 \\
\hline LV e' mean velocity $(\mathrm{cm} / \mathrm{s})$ & $8.5[7.0,10.8]$ & $9.3[7.8,11.0]$ & $9.0[7.0,10.5]$ & 0.33 \\
\hline E/e' average & $7.9[6.6,10.3]$ & $8.3[6.7,9.9]$ & $8.1[6.8,10.8]$ & 0.81 \\
\hline$E / A$ & $1.1[0.8,1.4]$ & $1.1[0.8,1.3]$ & $1.0[0.8,1.3]$ & 0.83 \\
\hline
\end{tabular}

Abbreviations: $\mathrm{A}=$ late transmitral flow; $\mathrm{E}=$ early transmitral flow; $\mathrm{E} / \mathrm{A}=$ ratio of early and late transmitral flows, diastolic function index; $E / e^{\prime}=$ early transmitral inflow divided by the average of the septal and lateral tissue Doppler indices e', diastolic function index; LV = Left Ventricle; LVEF = Left Ventricular Ejection Fraction.

† P-values represent differences in values between all three-time points tested with the Kruskal-Wallis test.

‡ Differences in values between prior to RT (T0) and immediately after RT (T1) were statistically significant $(p<0.05)$ according to the Wilcoxon signed-rank test.

** Differences in values between prior to RT (T0) and 5-9 months after RT (T2) were statistically significant $(p<0.05)$ according to the Wilcoxon signed-rank test 
Table 3 - Associations between the Rate of Change in Longitudinal Strain, Circumferential Strain, LVEF and E/e' with Mean Heart Dose $e^{\star \star}$

\begin{tabular}{|c|c|c|c|c|c|c|c|c|c|c|c|c|c|}
\hline & & \multicolumn{3}{|c|}{ LVEF } & \multicolumn{3}{|c|}{ Longitudinal strain } & \multicolumn{3}{|c|}{ Circumferential Strain } & \multicolumn{3}{|c|}{$E / e^{\prime}$} \\
\hline & & $\begin{array}{l}\text { LVEF (\%) } \\
\text { change per } \\
30 \text {-day } \\
\text { interval for } \\
\text { every } 100 \\
\text { cGy } \\
\text { exposure }\end{array}$ & $\begin{array}{l}(95 \% \\
\mathrm{Cl})\end{array}$ & $\begin{array}{l}P \\
\text { value }\end{array}$ & $\begin{array}{l}\text { Longitudinal } \\
\text { strain (\%) } \\
\text { change per } \\
\text { 30-day } \\
\text { interval for } \\
\text { every } 100 \\
\text { cGy } \\
\text { exposure }\end{array}$ & $\begin{array}{l}(95 \% \\
\mathrm{Cl})\end{array}$ & $\begin{array}{l}P \\
\text { value }\end{array}$ & $\begin{array}{l}\text { Circumferential } \\
\text { strain (\%) } \\
\text { change per } 30- \\
\text { day interval for } \\
\text { every } 100 \text { cGy } \\
\text { exposure }\end{array}$ & $\begin{array}{l}(95 \% \\
\mathrm{Cl})\end{array}$ & $\begin{array}{l}P \\
\text { value }\end{array}$ & $\begin{array}{l}\text { E/e' } \\
\text { change } \\
\text { per } 30- \\
\text { day } \\
\text { interval } \\
\text { for every } \\
100 \text { cGy } \\
\text { exposure }\end{array}$ & $\begin{array}{l}(95 \% \\
\mathrm{Cl})\end{array}$ & $\begin{array}{l}\mathrm{P} \\
\text { value }\end{array}$ \\
\hline \multirow{3}{*}{$\begin{array}{l}\text { Acute } \\
\text { change } \\
\text { Pre RT (T0) } \\
\rightarrow \\
\text { Immediately } \\
\text { after RT } \\
\text { (T1) }\end{array}$} & $\begin{array}{l}\text { Unadjusted } \\
\text { (Model 1) }\end{array}$ & -0.16 & $\begin{array}{l}(-0.42 \\
0.11)\end{array}$ & 0.26 & 0.08 & $\begin{array}{l}(-0.14, \\
0.31)\end{array}$ & 0.45 & -0.23 & $\begin{array}{l}(-0.70 \\
0.23)\end{array}$ & 0.32 & 0.13 & $\begin{array}{l}(-0.10 \\
0.36)\end{array}$ & 0.27 \\
\hline & $\begin{array}{l}\text { Minimally } \\
\text { adjusted } \\
\text { (Model 2) }\end{array}$ & -0.12 & $\begin{array}{l}(-0.33 \\
0.10)\end{array}$ & 0.29 & 0.15 & $\begin{array}{l}(-0.04 \\
0.34)\end{array}$ & 0.12 & -0.20 & $\begin{array}{l}(-0.59 \\
0.19)\end{array}$ & 0.31 & $0.13^{\S}$ & $\begin{array}{l}(-0.10 \\
0.35)\end{array}$ & 0.27 \\
\hline & $\begin{array}{l}\text { Fully } \\
\text { adjusted } \\
\text { (Model 3) }\end{array}$ & -0.22 & $\begin{array}{l}(-0.44 \\
0.01)\end{array}$ & 0.06 & 0.19 & $\begin{array}{l}(-0.01 \\
0.39)\end{array}$ & 0.06 & -0.18 & $\begin{array}{l}(-0.57 \\
0.20)\end{array}$ & 0.36 & $0.14^{\S}$ & $\begin{array}{l}(-0.09 \\
0.36)\end{array}$ & 0.23 \\
\hline \multirow{3}{*}{$\begin{array}{l}\text { Subacute } \\
\text { change } \\
\text { Pre RT (T0) } \\
\text { to 5-9 } \\
\text { months after } \\
\text { RT (T2) }\end{array}$} & $\begin{array}{l}\text { Unadjusted } \\
\text { (Model 1) }\end{array}$ & -0.02 & $\begin{array}{l}(-0.12 \\
0.08)\end{array}$ & 0.70 & 0.04 & $\begin{array}{l}(-0.04 \\
0.20)\end{array}$ & 0.30 & 0.07 & $\begin{array}{l}(-0.07 \\
0.20)\end{array}$ & 0.33 & 0.07 & $\begin{array}{l}(0.001 \\
0.13)\end{array}$ & 0.05 \\
\hline & $\begin{array}{l}\text { Minimally } \\
\text { adjusted } \\
\text { (Model 2) }\end{array}$ & -0.03 & $\begin{array}{l}(-0.13 \\
0.07)\end{array}$ & 0.54 & 0.05 & $\begin{array}{l}(-0.03 \\
0.13)\end{array}$ & 0.22 & 0.08 & $\begin{array}{l}(-0.05 \\
0.22)\end{array}$ & 0.24 & 0.06 & $\begin{array}{l}(- \\
0.002, \\
0.12)\end{array}$ & 0.06 \\
\hline & $\begin{array}{l}\text { Fully } \\
\text { adjusted } \\
\text { (Model 3) }\end{array}$ & -0.04 & $\begin{array}{l}(-0.14 \\
0.06)\end{array}$ & 0.44 & 0.05 & $\begin{array}{l}(-0.03 \\
0.13)\end{array}$ & 0.19 & 0.09 & $\begin{array}{l}(-0.05 \\
0.22)\end{array}$ & 0.22 & 0.06 & $\begin{array}{l}(- \\
0.005 \\
0.12)\end{array}$ & 0.07 \\
\hline
\end{tabular}

${ }^{\star *}$ Beta coefficients, 95\% Confidence Interval (Cl) are reported as per each 100 cGy exposure over 30 days; all changes represent absolute differences.

The unadjusted model (Model 1) included the echocardiographic variable of interest over time as our outcome measure, MHD, time from radiation treatment, and time by treatment interaction with MHD as our primary variable of interest.. The minimally adjusted model (model 2) included the variables in Model 1 in addition to adjusting for the baseline (T0) echocardiographic variable, age, race, pre-existing cardiovascular disease, as well as anthracycline and/or trastuzumab exposure prior to RT. The fully adjusted model (Model 3) included the variables in models 1,2 and an interaction term with anthracycline or trastuzumab exposure and MHD

$\S$ Due to issues of collinearity, E/e' models for acute changes were not adjusted for baseline E/e' in Models 2 and 3

Abbreviations: $\mathrm{Cl}=$ Confidence Interval; E/e' = diastolic function index; LVEF = left ventricular ejection fraction; MHD = Mean Heart Dose; RT = Radiation Therapy 

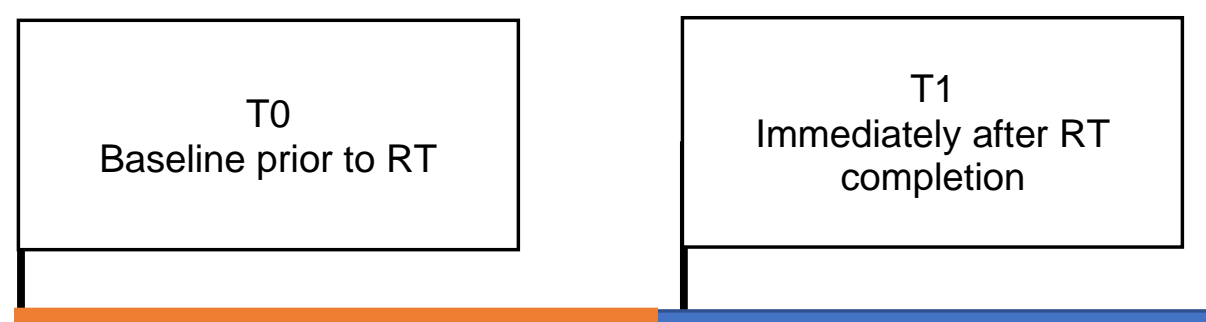

T2

5-9 months after RT completion

Radiation Therapy

8-week course

Figure 1 - $X X X(X X X X)$ study design 

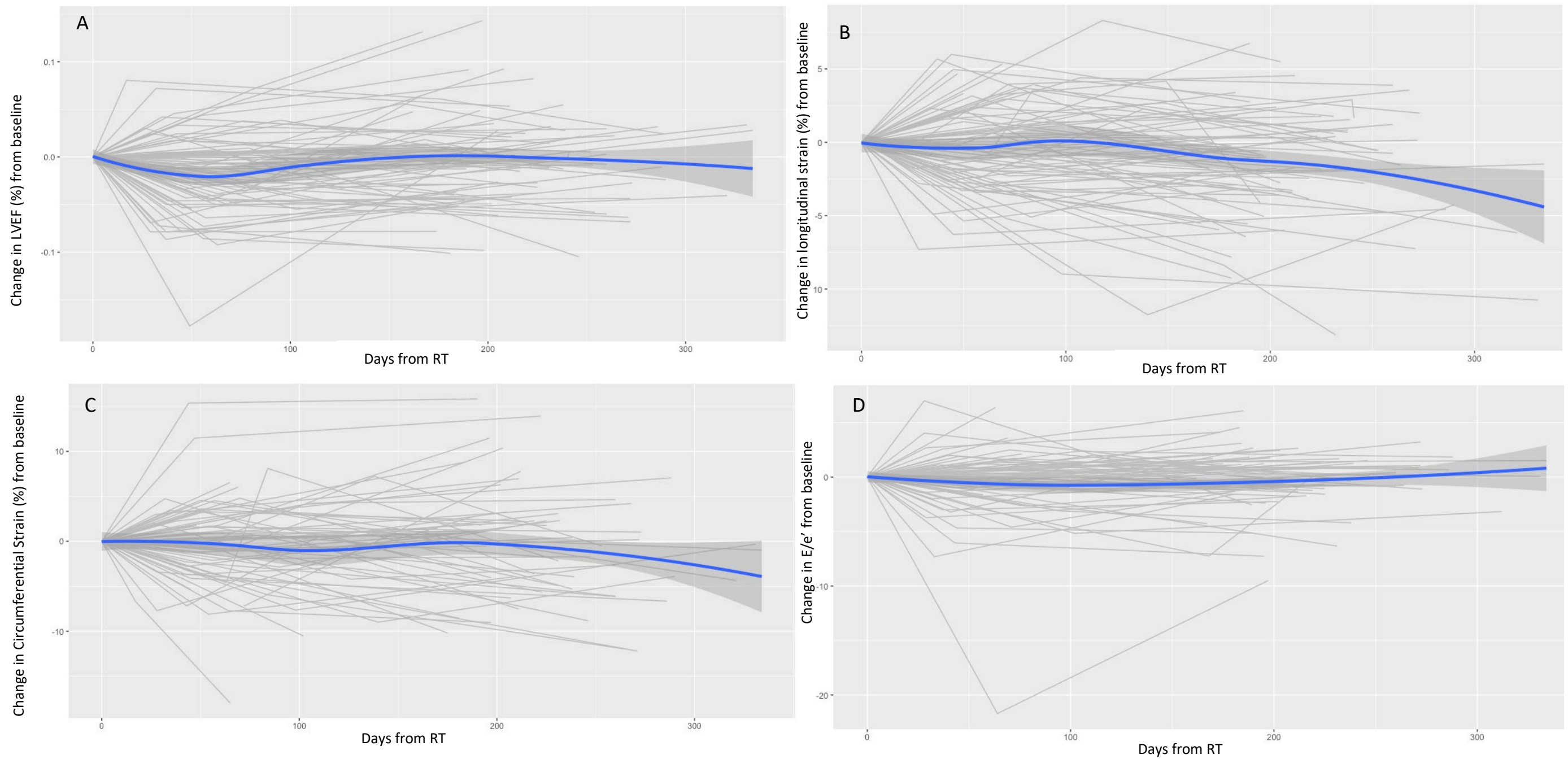

Figure 2 - Changes in echocardiographic measures (LVEF, longitudinal strain, circumferential strain, and E/e') from baseline values after RT therapy. 